\title{
A Note on the Electronic Computer at Rothamsted
}

The "401" experimental electronic computer built by Elliott Brothers under contract from the National Research Development Corporation is now housed in the Statistical Department at Rothamsted, machine time being shared on a 50-50 basis between the N.R.D.C. and the Department. The Department is applying the computer to problems which arise in statistical research and its applications to agriculture, but time on the machine is available through the N.R.D.C. for general computations. The use of the computer specifically for the former work is a new venture. Prior to Rothamsted's acquiring the 401 little was known of the advantages and difficulties of applying electronic computers to statistics. Consequently, before being able to take full advantage of this powerful aid to computation, it was necessary to obtain experience not only of the appropriate programming techniques but also of the methods of adapting the rather special numerical techniques. By using a prototype computer the relevant experience is being gained and at the same time the characteristics that a permanent machine in this field ought to have are becoming apparent. This note gives a short description of the machine and an indication of the type of work being carried out.

The 401 is a serial computer with a word length of 32 binary digits and a word time of $100 \mu$ s. The main store is a magnetic disc of 23 tracks, each holding 128 words; 7 of the tracks are always immediately available to the computer, but only one of the remaining 16 is accessible at any given time, switching between these tracks being by means of high-speed relays. There are 5 single word immediate-access registers; one is the accumulator which can be coupled with the second register for double length working; the other 3 registers can each be used to modify orders as well as for temporary storage of numbers. Input is by 5-hole punched tape and output by either electrical typewriter or teleprinter-punch; punch-card input is shortly to be incorporated. The machine has a two-address code to allow for optimum programming. Operations possible include addition, subtraction, multiplication with and without round off, collation, non-equivalence, discrimination on zero or negative values, and left and right shifting of single or double length numbers. Addition and subtraction take one word time $(100 \mu s)$ to carry out, a shift of $n$ places takes $n$ word times and multiplication 32 word times (3.2 m.sec.).

A library of sub-routines is being built up and many of the standard functions and operations are now available. Routines for analysing randomised blocks and Latin square experiments have been used to obtain results for several large series of experiments. Work on routines for the analysis of factorial experiments is under way-these will allow a fast and accurate assessment of a wide variety of experiments. A programme has been developed which calculates the means, standard deviations, variances, covariances and correlation coefficients for samples of multivariate data. Specific problems tackled include a sampling investigation of the "combination of probabilities" test of significance: this necessitated a very large amount of computation and has been successfully concluded. General routines developed include a "programming" routine and a set of routines for floating binary operations. The former inserts the appropriate addresses in a skeleton programme and punches out the final routine for immediate use, thus 
saving a great deal of programmer's time. The latter allows for the full 9 significant decimal figure accuracy and can accommodate numbers in the range $10^{ \pm 8000}$.

The encouraging start made at Rothamsted seems to indicate that electronic computers are well suited for dealing with statistical problems, but undoubtedly new numerical and programming techniques are necessary to meet the special requirements of such work.

Rothamsted Experimental Station

S. LIPTON

Harpenden

Herts., England

\section{REVIEWS AND DESCRIPTIONS OF TABLES AND BOOKS}

This section contains reviews and descriptions of tables and of publications which in some explicit way have general interest in connection with tables or computation. Those noted as "Deposited in the UMT File" have been deposited in the Unpublished Mathematical Tables file maintained by the Chairman of the Editorial Committee; they are available there for reference. In many cases the author of these tables has a limited number of copies available for distribution. Authors of works containing mathematical tables of general interest or which otherwise fall into the classes noted or reviewed here are urged to submit a copy to the Chairman of the Editorial Committee.

31[A, F].-RSMTC Table of Binomial Coefficients. Royal Soc. Math. Tables, v. 3. Edited by J. C. P. Miller. Cambridge 1954. viii + 162 p. $21.5 \times 28.0$ $\mathrm{cm}$. Price $\$ 6.50$.

This table gives exact values of binomial coefficients

$$
\left(\begin{array}{l}
n \\
k
\end{array}\right)=\frac{n !}{k !(n-k) !}
$$

for positive integer values of $k$ and $n$. The main table (p. 2-103) is for $n \leq 200$. Because

$$
\left(\begin{array}{l}
n \\
k
\end{array}\right)=\left(\begin{array}{c}
n \\
n-k
\end{array}\right)
$$

it is unnecessary to print values for $k>n / 2$. Actually $k$ is taken less than or equal to $(n+1) / 2$ plus whatever additional values of $k$ fill out the line. The central binomial coefficient in case $n$ is even, or the two central coefficients in case $n$ is odd, are printed in bold face type.

The rest of the table is for $n \leq 5000$ but for $k \leq L$ limited as follows

$\begin{array}{cr}n & L \\ 200-500 & 12 \\ 500-1000 & 11 \\ 1000-2000 & 5 \\ 2000-5000 & 3\end{array}$

